\title{
EPIDURAL BLOCK FOR TREATMENT OF RENAL COLIC DURING PREGNANCY
}

\author{
L. BRIAN READY AND ERIC S. JOHNSON
}

ABSTRACT

This report documents for the first time the application of continuous segmental epidural block ( $\mathrm{T} 11$ to $\mathrm{L} 2$ ) to treat renal colic complicating pregnancy. The block was maintained for 16 hours and the pain did not recurr. Advantages for the mother, and possibly for the foetus, are considered. The published experience of others using this technique in nonpregnant patients is reviewed.

Key Words: Anaesthesia, Epidural Block; Pregnancy, renal colic.

THE REPORTED INCIDENCE of ureteral calculi occurring during pregnancy varies widely. Strong, Murchison and Lynch found that in a time period when 22,495 deliveries were performed, 14 pregnant patients were hospitalized for treatment of urinary calculi - an incidence of one in 1,600 deliveries $\left(0.062\right.$ per cent). ${ }^{1}$

Although uncommon, ureteral calculi complicating pregnancy are said to represent the largest group of painful conditions occurring during pregnancy that require hospitalization. Compression of ureters by the gravid uterus appears to result in dilatation and urinary stasis. ${ }^{2}$ These factors may predispose the pregnant patient to urolithiasis.

Conservative management of pregnant patients with ureteric calculi consists primarily of hydration and systemic analgesics. The use of continuous segmental epidural blockade, so popular for relieving the pain of childbirth, has not been reported as a therapeutic measure in the treatment of ureteric calculi that occur during pregnancy. The following case report documents such an application of the technique.

\section{CASE REPORT}

A 31-year-old gravida I patient with a 23-week intrauterine pregnancy was admitted to the hospital with intermittent right flank pain, nausea, anorexia, and urinary frequency for five days.

L. Brian Ready, M.D., F.R.C.P.(C), Assistant Professor; Eric S. Johnson, M.D., Research Fellow; Department of Anesthesiology, RN-10, University of Washington School of Medicine, Seattle, Washington 98195, U.S.A.

Reprint requests and correspondence to Dr. L. Brian Ready.
She denied dysuria, fever, and chills, and there was no history of previous urinary tract infection or urolithiasis. Abdominal examination elicited marked right flank tenderness on deep palpation. The following laboratory data were obtained: haematocrit 43, WBC 17,900 (polymorphonuclear 15,200 , bands 800 , lymphocytes 1,610 , monocytes 980 ); urinalysis $\mathrm{RBC} 1+$, bacteria $\mathrm{l}+$, no WBC; urine culture grew mixed flora. Though no renal or ureteral calculi were identified on a preliminary abdominal radiograph, an excretory urogram (a single $\mathrm{X}$-ray at 10 minutes) demonstrated an intensified nephrogram on the right side with delayed appearance of dye in the right pyelocalyceal system and dilatation of the calyces (Figure 1). The diagnosis was right ureteral calculus obstructing the right ureter.

The pain was poorly controlled by parenteral narcotic analgesics. Persistent nausea and emesis necessitated intravenous fluids. The patient's symptoms were unchanged by the end of the second day of hospitalization and she was bedridden.

Permission for a trial of epidural anaesthesia was obtained from the patient. She was transferred to the labour and delivery area where the nursing personnel are familiar with this type of regional anaesthetic. She received a preload of $500 \mathrm{ml}$ of lactated Ringer's solution intravenously. With the patient in a lateral position, and under aseptic conditions, an 18 gauge Crawford needle was placed into the epidural space from a paramedian approach at the L2 interspace. A catheter was threated $4 \mathrm{~cm}$ cephalad. Following removal of the needle, no blood or CSF could be aspirated through the catheter. A two ml test dose of 2-chloroprocaine 3 per cent produced no discernable sensory change. An additional $2 \mathrm{ml}$ of 


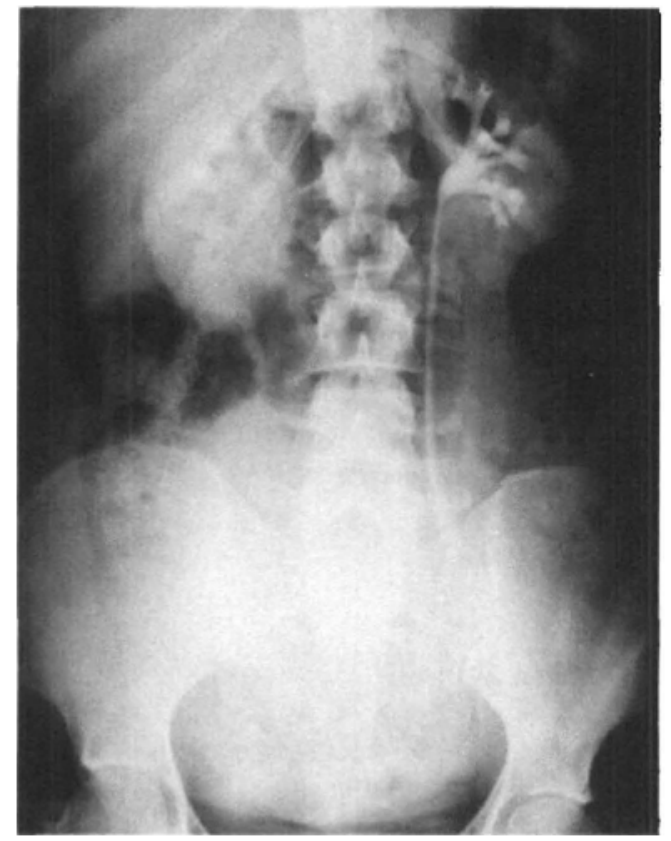

Figure 1 Ten minute excretory urogram demonstrating intensified nephrogram on the right side with delayed appearance of dye in the right pyelocalyceal system and dilatation of the calyces.

2-chloroprocaine 3 per cent resulted in a sensory loss limited to an area between dermatomes T11 and $\mathbf{L} 2$ bilaterally. The patient was completely free of pain within 10 minutes. She required no additional analgesics and, within three hours of the initiation of the anesthetic, she was tolerating oral fluids well, was voiding, and was ambulatory with standby assistance. The epidural block was maintained by injection of $3 \mathrm{ml}$ of bupivicaine 0.5 per cent through the catheter every two to three hours for 16 hours.

The pain did not recur following the 8 th injection, and though no stone was found in her screened urine, it was felt the stone had passed from the ureter. The epidural catheter was removed and the patient was discharged from the hospital pain-free. Seventeen weeks later, she underwent caesarean section under epidural anesthesia for a Frank breech and arrest of dilatation and descent. A healthy male infant was delivered.

\section{Discussion}

The ureters are innervated through the renal, ovarian (or spermatic) and hypogastric plexuses which contain sympathetic, parasympathetic and sensory fibers. The sensory (pain) fibers are components of the 11 th and 12 th thoracic and 1st lumbar nerves. ${ }^{3}$ An obstructing calculus in the renal pelvis or ureter causes stimulation of sensory nerve endings, primarily as a result of distention of the renal pelvis. An additional source of noxious stimulation is reflex spasm of the smooth muscles of the ureteropelvic junction and ureters. The resulting renal colic can be one of the most painful conditions known and is frequently not relieved adequately even with potent narcotic analgesics. Further, this group of drugs can be associated with distressing side effects, such as the nausea and vomiting which occurred in our patient before the epidural block. Narcotics may also interfere with normal peristalsis in ureteric smooth muscle.

Severe pain is known to result in a stress response that includes the outpouring of endogenous catecholamines. During pregnancy, it might be speculated that this situation would compromise uterine blood flow. ${ }^{4}$ Thus, besides the compelling humanitarian reasons for wishing to relieve severe pain in all patients, the well-being of the foetus may be an additional reason for wishing to ensure good maternal analgesia during pregnancy.

Bonica has reported a number of cases of successful treatment of renal colic with regional anaesthetic techniques. ${ }^{5} \mathrm{He}$ employed unilateral paravertebral sympathetic blocks from $\mathrm{T} 10$ to $\mathrm{L} 2$ in some patients and continuous segmental epidural blockage in others. He noted advantages of continuous epidural blockade which included the ability to maintain blockade over an extended period of time and the ability to extend the blockade to provide anaesthesia for a cystoscopy, basket extraction of a calculus, or open surgery when those procedures became necessary.

It is possible that regional analgesia, in addition to providing pain relief, may also fayorably influence the natural course of the disease process associated with ureteric calculi. Helstrom ${ }^{6}$ observed that in 275 non-pregnant patients presenting with renal colic and treated only with systemic analgesics, 23 per cent spontaneously passed their stones within three days of hospital admission. Lloyd and Carey ${ }^{7}$ reported that in a series of 11 non-pregnant patients treated with a combination of narcotics and epidural blockade, 55 per cent passed stones spontaneously within the same time period. In a series of 22 nonpregnant patients treated only with continuous epidural block reported by Romagnoli and Batra ${ }^{8}$ $\mathbf{7 2 . 7}$ per cent passed renal calculi spontaneously. The duration of block in their series ranged from 
18 to 42 hours with an average of 32.4 hours. The studies quoted are not directly comparable, since a number of important variables were not standardized. These include the hydration protocols used and the use or omission of narcotics. In the case of the series reported by Romagnoli and Batra, the number of cases studied was small. However, it is interesting to note that over 70 per cent of their patients treated with continuous segmental epidural blockade and vigorous hydration did pass their stones spontaneously.

Continuous segmental epidural blockade is already widely practiced in modern obstetrical units to provide pain relief for labour and delivery. The safety of this technique for mother and foetus, when practiced with skill, is now wellestablished. Nursing personnel on these units are familiar with the care of patients with an established block. If an epidural block is carefully confined to only the necessary segments for pain relief ( $\mathrm{T} 11$ to $\mathrm{L} 1$ ), many patients will be able to move about with assistance and most will be able to void without difficulty. Women who are completely comfortable and who are able to move with ease will be at less risk of thromboembolic phenomena than those who are immobile in bed with severe pain.

In summary, a patient presenting during pregnancy with renal colic was treated with continuous segmental epidural block. Compared to standard conservative treatment with narcotic analgesics, segmental blockade resulted in a totally pain-free patient who suffered no nausea and vomiting, was able to walk about and apparently passed her calculus. It is currently not known whether treatment of renal colic with continuous segmental epidural block improves the probability of spontaneous passage of a renal stone. Whether or not this proves to be the case, we feel that this technique offers significant advantages over other forms of conservative therapy. In view of these advantages and the safety of the technique, when performed by practitioners expert in epidural anesthesia in a setting where adequate nursing supervision is possible, we recommend it as the conservative treatment of choice in all patients who present with renal colic. There may be special benefits when patients present during pregnancy.

\section{REFERENCES}

1. Strong, D.W., Murchison, R.J. \& LYNCH, D.F. The management of ureteral calculi during pregnancy, Surgery, Gynecology and Obstetrics. 146: 604-608 (1978).

2. Hendricks, C.H. \& Barnes, A.C. Effects of supine position on urinary output in pregnancy, Amer. J. Obstet. \& Gynec. 69: 1225-1232 (1955).

3. BonicA, J.J. The Management of Pain. Lea and Febiger. p. 1404 (1953).

4. Shnider, S.M. \& Levinson, G. Anesthesia for Obstetrics. Williams and Wilkins. p. 31 (1979).

5. Bonica, J.J. The Management of Pain. Lea and Febiger. p. 1405-1407 (1953).

6. Helstrom, J. Aetiological and therapeutic experiences concerning kidney and ureteric stones, Brit. J. Urol. $21: 9$ (1949).

7. Lloyd, J.W. \& Carrie, L.E.S. A method of treating renal colic, Proc. R. Soc. Med. 58: 634 (1965).

8. Romagnoli, A. \& Batra, M.S. Continuous epidural block in the treatment of impacted ureteric stones. Can. Med. Assoc. J. 109: 968 (1973).

\section{RÉSUMÉ}

Cette observation décrit pour la première fois l'application d'un bloquage épidural continu segmentaire ( $\mathrm{T} 11$ à $\mathrm{L} 1$ ) au traitement de la colique rénale pendant la grossesse. On a maintenu le bloquage pendant 16 heures sans réapparition de la douleur. Les avantages pour la mère et possiblement pour le foetus sont discutés. Les auteurs font un tout d'horizon des publications concernant l'utilisation de cette technique chez des patientes chez qui la grossesse n'est pas un problème. 